\title{
Optimal asymmetry of transistor-based terahertz detectors
}

\author{
Aleksandr Shabanov, ${ }^{1}$ Maxim Moskotin, ${ }^{1}$ Vsevolod Belosevich, ${ }^{2,3}$ Yakov Matyushkin, ${ }^{1,3}$ Maxim Rybin, ${ }^{4}$ Georgy \\ Fedorov, ${ }^{1}$ and Dmitry Svintsov ${ }^{1}$ \\ 1) Moscow Institute of Physics and Technology, Dolgoprudny 141700, Russia \\ ${ }^{2)}$ Physics Department, Moscow State Pedagogical University, Moscow 119435, \\ Russia \\ 3) National Research University Higher School of Economics, Moscow 101000, \\ Russia \\ 4) Prokhorov General Physics Institute of the Russian Academy of Sciences, Moscow 119991, \\ Russia
}

Detectors of terahertz radiation based on field-effect transistors (FETs) are among most promising candidates for low-noise passive signal rectification both in imaging systems and wireless communications. However, it was not realised so far that geometric asymmetry of common FET with respect to source-drain interchange is a strong objective to photovoltage harvesting. Here, we break the traditional scheme and reveal the optimally-asymmetric FET structure providing the maximization of $\mathrm{THz}$ responsivity. We fabricate a series of graphene transistors with variable top gate position with respect to mid-channel, and compare their sub$\mathrm{THz}$ responsivities in a wide range of carrier densities. We show that responsivity is maximized for input gate electrode shifted toward the source contact. Theoretical simulations show that for large channel resistance, exceeding the gate impedance, such recipe for responsivity maximisation is universal, and holds for both resistive self-mixing and photo-thermoelectric detection pathways. In the limiting case of small channel resistance, the thermoelectric and self-mixing voltages react differently upon changing the asymmetry, which may serve to disentangle the origin of nonlinearities in novel materials.

Sensitive detection of sub-terahertz and terahertz $(\mathrm{THz})$ radiation is vital for security applications, defect inspection, radio-astronomy, and medical imaging ${ }^{1}$. Currently, the main application niche for sub- $\mathrm{THz}$ detectors is wireless communications, where increase in carrier frequency promises a proportional increase in data transfer rates 2 . Recently, antenna-coupled field-effect transistors (FETs) have emerged as prospective candidates for passive sub- $\mathrm{THz}$ and $\mathrm{THz}$ detection ${ }^{3-5}$. Compared to active systems based on high-frequency amplifiers and followed by rectifiers, passive FET-based systems feature low power consumption and can operate above the cutoff frequency $\underline{\underline{6}}$. Compared to passive systems based on diodes $^{7}$, FETs are compatible with planar CMOS technology and do not require complex three-dimensional designs. The presence of extra control electrode in FETs simplifies the phase coherent (homodyne ${ }^{8}$ and heterodyne $^{-}$) detection. These advantages stimulate a booming research on $\mathrm{THz}$ FET detectors, including those based on novel materials $10-14$, alternative mechanisms of current manipulation ${ }^{15,16}$, and exploiting plasmonic effects ${ }^{17}=19$.

It has been scarcely realised that most FETs operating as $\mathrm{THz}$ detectors have an intrinsic deficiency lying in symmetry of the structure with respect to the interchange of source and drain. Once the signal is fed between source and drain, the photovoltage may build up only at finite bias ${ }^{20,21}$, which results in large Flicker noise. Finite zero-bias response for source-drain signal coupling appears also if a lateral $p-n$ junction is introduced in the channel which, however, requires extra "doping gates" 22 . Coupling the $\mathrm{THz}$ signal between source and gate, known as Dyakonov-Shur scheme ${ }^{23}$, introduces an asymmetry of signal feeding, and thus results in the desired zero-bias photovoltage between source and drain ${ }^{24}$.

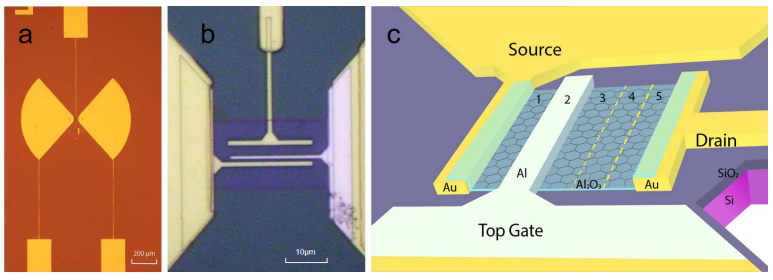

FIG. 1. Antenna-coupled graphene FET as terahertz detector (a) Optical image of the antenna-coupled device. Scale bar is $200 \mu \mathrm{m}$ (b) Zoomed-in photograph of the gated channel. Scale bar is $10 \mu \mathrm{m}$. (c) Schematic of the FET-based detector with asymmetric gate location. Numbers indicate five gate positions used in our study.

To date, most research on THz FETs blindly copied the proposal of Dyakonov and Shur with geometrically symmetric channel10,11,24-27. It was not attempted to enhance the responsivity by introducing extra geometric asymmetry that is achieved, most simply, by displacement of antenna-coupled gate electrode away from midchannel. The necessity for geometric asymmetry was recently realised for $\mathrm{THz}$ emission applications $\frac{28,29}{}$, but its role in detection process remain largely unexplored.

In this paper, we find an optimal geometric asymmetry of a FET-based antenna-coupled detector of $\mathrm{THz}$ radiation. We fabricate a series of graphene-based transistors with variable position of signal gate (Fig. 1). Measurements of sub- $\mathrm{THz}$ photoresponse at room and liquid nitrogen temperatures show $\sim 5$-fold responsivity enhancement of a structure with proximized source and gate, compared to that with proximized drain and gate.

We substantiate the obtained results theoretically, assuming two common rectification mechanisms: photo- 



FIG. 2. (a) Dependence of structures' channel resistance on bottom gate voltage for devices with different gate position. All curves are shifted such that charge neutrality voltages $V_{\text {CNP }}$ coincide. (b) Dependence of photovoltage on bottom gate voltage, obtained for the same devices upon illumination with sub- $\mathrm{THz}$ radiation $(f=130 \mathrm{GHz})$. (c) Dependence of photovoltage on top gate voltage for the same devices at temperature of liquid nitrogen. (d) Photovoltage swing upon variation of charge carrier density vs gate position obtained at $T=77 \mathrm{~K}$ and $T=300 \mathrm{~K}$

thermoelectric (Seebeck) effect at metal-graphene junctions $\frac{30,31}{31}$ and resistive self-mixing in the gated channe10,32. Once the graphene channel resistance $R_{\mathrm{ch}}$ exceeds the ac gate impedance $\left|Z_{C}\right|$, both rectification channels benefit from source-shifted asymmetry, in accordance with experimental data. Our result has a simple interpretation if one assumes thermoelectric origin of photoresponse: co-location of source and gate increases the power density released at source metal-graphene junction, and enhances the average thermal gradient across the structure. The trend for resistive self-mixing does not admit that simple explanation, and actually changes for small $R_{\mathrm{ch}}$. In the limiting case $\left|Z_{C}\right| \gg R_{\mathrm{ch}}$, the self-mixing signal is reduced with shifting the gate toward the source, while Seebeck signal stays roughly constant. Thus, experiments with variable gate location can shed light on dominant $\mathrm{THz}$ rectification pathways in graphene $\mathrm{e}^{25,26}$ and emerging $2 \mathrm{D}$ materials $\frac{11}{}$.

The key challenge toward the quantitative comparison of photoresponse in CVD-graphene FETs lies in fluctuations of characteristics from one device to another. To circumvent this, we have fabricated nominally identical devices from a single millimetre-scale layer of graphene. It was grown in a home-made cold-wall reactor on copper foil ${ }^{38,39}$. Grapene was wet-transferred onto an oxidized $\mathrm{Si}$ substrate with $\mathrm{SiO}_{2}$ dielectric of thickness $d_{b}=500 \mathrm{~nm} \underline{40}$. SLG FET channel was patterned to a rectangular shape with length $L=4 \mu \mathrm{m}$ and width $W=20 \mu \mathrm{m}$ by e-beam lithography (PMMA mask) and etching in $\mathrm{O}_{2}$ plasma and then supplied with two small metal contacts. These source and drain electrodes were made of gold $(40 \mathrm{~nm})$ with vanadium adhesion layer $(5 \mathrm{~nm})$. To maintain stability of graphene channel with respect to atmospheric contaminants, an aluminum oxide cover was deposited using electron-beam evaporation with a thickness of $d_{t}=100 \mathrm{~nm}$ and lift-off technique, which would also serve as a top gate dielectric. The small source electrode was coupled by a sleeve of a dipole antenna, and the other sleeve was deposited over the top gate [Fig. 1 $(a, b)]$. The top gate for various FETs was placed in five sequential positions, from that proximized to the source (labelled as \#1) to that in immediate vicinity to the drain (labeled as \#5) [Fig. [1 (c)]. For each device, we have performed electrical DC characterization; the silicon substrate covered with $500 \mathrm{~nm}$ of silica was used as a back gate. The dependences of the sample resistance on the gate voltage are presented in Fig. 2(a); for all devices there is a maximum resistivity corresponding to the charge neutrality point (CNP) of graphene.

The main experiment consists in measurements of photovoltage $V_{\mathrm{p}}$ generated in FETs upon their illumination with sub-THz radiation of frequency $f=130 \mathrm{GHz}$. In this experiment, the detector on substrate was mounted on a holder with silicon lens arranged in a cryostat. Radiation was fed from backward wave oscillator with power $P \sim 1 \mathrm{~mW}$ calibrated with Golay cell.

The results of photovoltage measurements for various gate locations are summarized in Fig. 2. The dependence of $V_{\mathrm{p}}$ on back gate voltage has a well-recognized anti-symmetric shape. Such shape is typical for both photo-thermoelectric and resistive self-mixing rectification pathways. A remarkable property of the recorded characteristics lies in their swing. Indeed, the photovoltage response for rightmost top gate position is faint and noisy, while the characteristic responsivity $R_{V}=V_{\mathrm{p}} / P$ hardly reaches $0.5 \mathrm{~V} / \mathrm{W}$. The swing gradually goes up upon shifting the gate to the source, and reaches its maximum value for top gate proximized to the source. The characteristic responsivity for such geometry already reaches $2.5 \mathrm{~V} / \mathrm{W}$, which is five times larger than for the rightmost gate position.

To provide a simple figure-of-merit for devices with various asymmetry, we compare their photovoltage swings $\Delta V_{\mathrm{p}}$ being the differences between maximum and minimum photovoltages recorded upon variation of carrier density, Fig. 2(d). Such a figure is insensitive to the unavoidable shifts of CNP due to large-scale doping fluctuations within CVD graphene layer. The maximum and 

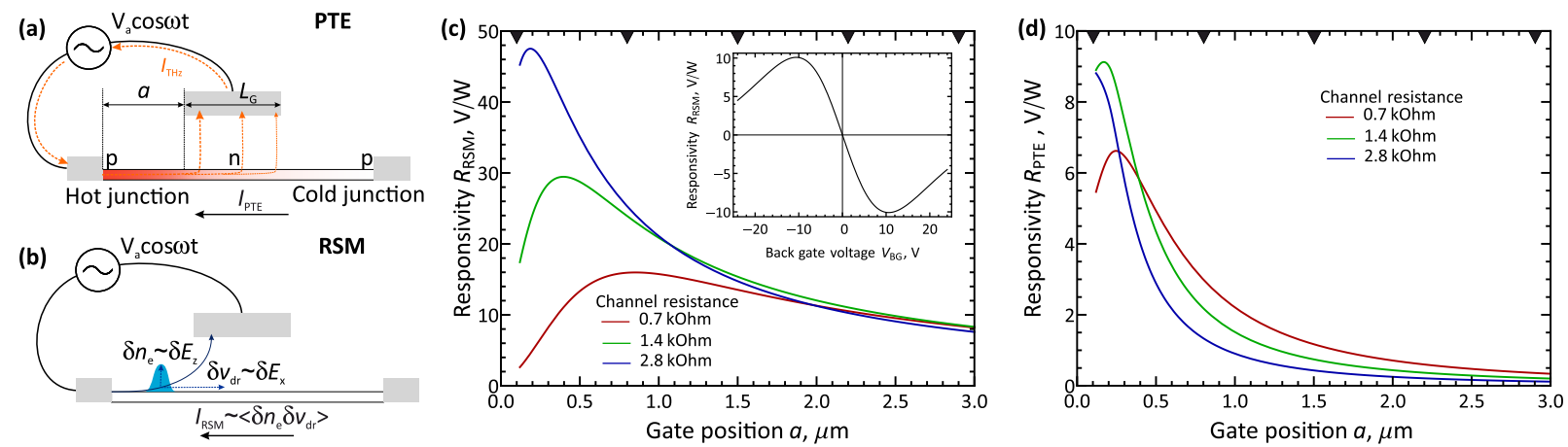

FIG. 3. (a,b) Schematic representation of photothermoelectric (a) and resistive self-mixing (b) mechanisms in FETs with antenna coupled between source and gate. In (a), asymmetric distribution of AC current $I_{\mathrm{THz}}$ leads to overheating of source side of the junction, and to the thermal diffusion current toward the source. In (b), the transverse component of $\mathrm{AC}$ electric field induces extra charge carriers $\delta n_{e}$ and enhances the channel conduction. These carriers are subsequently dragged by inplane component of the field. The time-averaged current $I_{\mathrm{RSM}}$ is directed toward the source. (c,d) Calculated responsivities of resistive self-mixing (c) and photo-thermoelectric effects in devices with variable gate-to-source separation $a$ calculated for different channel resistances $R_{\mathrm{ch}}$. All curves calculated for fixed Fermi energy $E_{F}=100 \mathrm{meV}$ and variable momentum relaxation times $\tau$ linked to resistivity $\rho$ via Drude formula $\rho=m\left(E_{F}\right) / n_{e} e^{2} \tau$. Inset in (c) shows the dependence of responsivity on back gate voltage for device with $a=0.5 \mu \mathrm{m}$.

minimum are reached at two sides of CNP, corresponding to weak hole/electron doping of the channel. Again, the trend toward increased photoresponse with shifting the gate toward the source is recognised in photovoltage swing data.

The trend toward increased photoresponse for sourceshifted feeding gate persists upon cooling the sample to liquid nitrogen temperature, as shown in Fig. 2 (c). The data for sample with gate position \# 1 fall out of this trend, probably due to sample-to-sample variations. Instructively, the characteristic responsivity at $T=77 \mathrm{~K}$ is almost the same as at $T=300 \mathrm{~K}$. Weak sensitivity of electrical properties to the temperature in CVD graphene may be explained by a large number of intrinsic defects 33 obtained during growth and wet transfer.

We now turn to theoretical interpretation of the data assuming the two most common rectification mechanisms in graphene channel, the photo-thermoelectric effect (PTE) and the resistive self-mixing (RSM), illustrated in Fig. 3 (a), (b). The PTE voltage emerges due to asymmetric heating of the device by ac electric current circulating between source and top gate 25,31 . The metal-graphene junction at the source is strongly heated, while the junction at the drain remains at lower electron temperature. The difference in thermoelectric voltages generated by hot and cold junctions results in overall finite photovoltage between source and drain. The RSM emerges upon induction of charge carriers in the channel by transverse component of ac electric field, and subsequent drag of induced carriers by the longitudinal component 32 .

Our quantitative model of photo-response is based on finding ac electric potential distribution $\varphi_{\omega}(x)$ in the channel induced by antenna voltage $V_{a} \cos \omega t=$ $\left(e^{-i \omega t}+e^{i \omega t}\right) V_{a} / 2$. This voltage can be bound to incoming THz power $P$ via antenna radiation resistance $Z_{\mathrm{rad}}$,
$P=V_{a}^{2} / 8 Z_{\mathrm{rad}}$ 34. In the gated section, the potential is governed by the telegrapher's equation

$$
\varphi_{\omega}+k^{2} \frac{\partial^{2} \varphi_{\omega}}{\partial x^{2}}=\frac{V_{a}}{2}
$$

where $k=(\sigma / i \omega C)^{1 / 2}$ is the wave vector of overdamped $2 \mathrm{~d}$ plasmons ${ }^{23}, \sigma$ is the conductivity of graphene sheet, and $C$ is the top gate-to-channel capacitance per unit area. The left ungated section of length $a$ is modelled as a lumped resistor, $\varphi_{\omega}(0)=a \varphi_{\omega}^{\prime}(0)$. The rightmost ungated section does not affect the photoresponse as almost no ac current is flowing into the drain, thus $\varphi_{\omega}^{\prime}\left(L_{g}\right)=0$. The geometric asymmetry introduced by shift of the top gate is now reflected in the asymmetry of the boundary conditions for electric oscillation problem.

The distribution of electric field $E_{\omega}(x)=-\partial \varphi_{\omega} / \partial x$ obtained from Eq. (11) is subsequently used to calculate the RSM and PTE photoresponse. The respective voltages $V_{\mathrm{RSM}}$ and $V_{\mathrm{PTE}}$ are given by $\stackrel{19,25}{\underline{1}}$

$$
\begin{gathered}
V_{\mathrm{RSM}}=2 \frac{d \sigma\left(V_{\mathrm{tg}}\right)}{d V_{\mathrm{tg}}} \frac{1}{C \omega} \operatorname{Im} \int_{0}^{L_{G}} E_{\omega}(x) \frac{\partial E_{\omega}^{*}(x)}{\partial x} d x, \\
V_{\mathrm{PTE}}=\left[S_{\mathrm{ch}}\left(V_{\mathrm{bg}}\right)-S_{\mathrm{cont}}\right]\left[T_{d}-T_{s}\right] .
\end{gathered}
$$

Above, $S_{\mathrm{ch}}\left(V_{\mathrm{bg}}\right)$ and $S_{\text {cont }}$ are the Seebeck coefficients in the back-gated channel and graphene in immediate contact with metal. For gold-contacted CVD graphene, the contacts are generally $p$-doped $\left(S_{\text {cont }}>0\right)$, which results in overall shift of all responsivity curves to negative photovoltages. $T_{s}$ and $T_{d}$ in Eq. (3) are the electron temperatures at the metal-induced p-n junctions in graphene located near the source and the drain, these are found by solving heat conduction equation with ac Joule heating 
as a source:

$$
\frac{\partial}{\partial x}\left(\chi_{e} \frac{\partial T}{\partial x}\right)-c_{e} \frac{T-T_{0}}{\tau_{\varepsilon}}=2 \sigma\left|E_{\omega}\right|^{2},
$$

above $c_{e}$ is the electronic heat capacitance, $\chi_{e}$ is the electronic thermal conductivity, and $\tau_{\varepsilon}$ is the energy relaxation time associated with supercollisions and emission of substrate phonons. We have assumed $\tau_{\varepsilon}=1 \mathrm{ps}^{41}$, the trends in PTE with gate shifts do not depend on specific value. Equation (4) was solved assuming fixed temperature $T_{0}$ (base cryostat temperature) at source and drain. The temperatures of interest $T_{s}$ and $T_{d}$ are evaluated at small distance $\delta L=125 \mathrm{~nm}$ from source an drain. This distance is the typical length of metal-induced $p-n$ junction acting as a thermoelectric generator 31 .

With the developed model, we have calculated the gate voltage dependences of photoresponse and found good qualitative agreement with experiment [inset in Fig. [3(c)]. Further on, we fixed numerically the value of carrier density in the channel and inspected the changes in photoresponse with varying the top gate position at various momentum relaxation times [Fig. 3(c), (d)]. Again, in good agreement with experimental data, the calculated photoresponse is growing as the position of the top gate is shifted toward the source for both rectification pathways. Remarkably, RSM and PTE photovoltages react differently on changes in the relaxation time. While RSM is weakly sensitive to $\tau_{p}$, the PTE signal benefits from increasing the momentum relaxation time.

In complement to the developed theory, it is possible to give a simple and intuitive interpretation of the measured data. The main dimensionless parameter governing the detector operation is the ratio of gate-tochannel capacitor impedance and gated channel resistance $p=\left[\omega C W L_{G} R_{G}\right]^{-1} \equiv k^{2} L_{G}^{2}$. Our detector operates in high-resistance mode wherein $p \ll 1$ (indeed, $R_{G} \sim 1 \mathrm{k} \Omega$ and $\left(\omega C L_{G} W\right)^{-1} \sim 100 \Omega$ in our device). Therefore, the $\mathrm{THz}$ voltage generated by the antenna drops mainly on graphene channel but not on the gatechannel capacitance. The power dissipated on ungated section $P \approx V_{a}^{2} / 2 R_{u}$ is growing as its length $a$ is decreased. The power density (per unit length) is increased even faster, $q \propto a^{-2}$ which results in an abrupt increase in junction heating and, hence, PTE voltage with shifting the gate to the source. The RSM effect should also benefit from left-ward shifting of the gate. Whence $k L \ll 1$, it is possible to estimate the longitudinal electric field under the gate as $V_{a} / a$. Reducing $a$ leads to stronger potential variations in the gated section and stronger self-mixing effects.

It is instructive that in the other limiting case of large gate-channel capacitance, $R_{\mathrm{ch}} \ll\left|Z_{C}\right|$, the behaviour of RSM and PTE effects with shifting the signal gate is very different (Fig. 4). In such a limit, the amplitude of current through graphene channel $I_{\mathrm{THz}}$ is fixed by large gate impedance. The power dissipated in the ungated section $P_{u} \approx 2\left|I_{\mathrm{THz}}\right|^{2} R_{u}$ is proportional to the ungated length $a$, while the power density setting the temper-
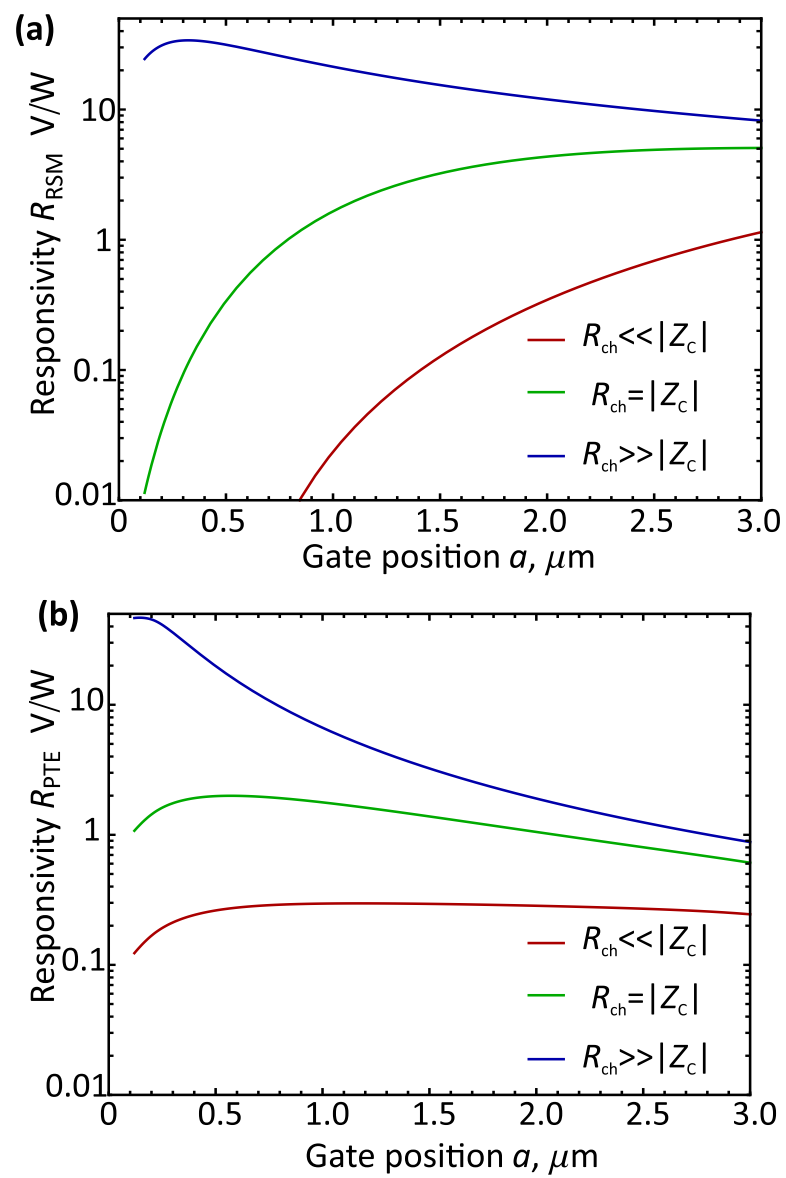

FIG. 4. Calculated dependences of resistive self-mixing (a) and photo-thermoelectric (b) responsivities on gate-to-source separation $a$ for various ratios of channel resistance $R_{\mathrm{ch}}$ and top gate capacitor impedance $Z_{C}=\left(i \omega C_{\mathrm{tg}}\right)^{-1}$. While for $R_{\mathrm{ch}} \gg\left|Z_{C}\right|$ both mechanisms are maximised at small $a$, the trend is reversed for $R_{\mathrm{ch}} \ll\left|Z_{C}\right|$. Blue and green curves plotted for $Z_{C}=100 \Omega$, red curve - for $Z_{C}=1 \mathrm{k} \Omega$. The value of $R_{\mathrm{ch}}$ is $100 \Omega$ for red and green curves, and $1.4 \mathrm{k} \Omega$ for blue curve.

ature is independent of $a$. The numerically calculated variations of PTE response in this limit with shifting the gate are slight and appear due to non-local character of heat transport equation. Contrary to the PTE, the RSM signal goes down with reducing $a$.

In principle, the experiment with variable gate position in the limit of large capacitive impedance may help to conclude whether the dominant $\mathrm{THz}$ detection mechanism in graphene is RSM or PTE ${ }^{25}$. Such problem can be hardly resolved with simple gate-dependtent measurements. Indeed, the dependences of both mechanisms on carrier density are indistinguishable and follow the derivative of conductivity with respect to Fermi energy. The experiment with variable gate position is also applicable to other 2d materials and heterostructure FETs, where the origins of photoresponse are debated ${ }^{11,14}$.

Our interpretation of data was based on specific boundary conditions for ac current and voltage at the 
FET terminals. These imply fixed voltage at the antenna-coupled terminals, and zero current at the drain. The latter condition is approximate, as it neglects the leakage current through the fringing capacitance between the gate and the drain $C_{g d} 27$. At the same time, this capacitance is reduced once we reduce the gate-drain separation $a$, and so does the leakage current. This may be another reason contributing to enhanced photoresponse of FETs with close source and gate.

To conclude, we have shown that the optimal asymmetric structure of antenna-coupled terahertz detecting FET is the one with source proximized to the feeding gate. Considering the FET as two rectifying contacts located at the source and drain junctions, we may say that placement of the gate near the source concentrates the electromagnetic energy at one of the rectifiers, leaving the other intact. Following such arguments, we may suggest that 'source-shifted asymmetry' of FET-based detectors would be beneficial also for rectification by hydrodynamic non-linearities of electron fluid ${ }^{23,35}$, non-linearities of Schottky $/ p-n$ junctions of the contacts 36 , and photocurrent generation by photovoltaic effect ${ }^{37}$.

Supplementary material. See supplementary material for resistance and photovoltage data in a broad range of gate voltages.

Acknowledgement. Experimental work of MM, VB, and GF was supported by grant \# 21-72-20050 of the Russian Science Foundation. YM, GF and VB acknowledge support of the RFBR grant \# 21-52-12041 (graphene post-synthesis processing and device design). Theoretical work of AS and DS was supported by grant \# 21-79-20225 of the Russian Science Foundation.

Data availability statement. The data that support the findings of this study are available from the corresponding author upon reasonable request.

${ }^{1} \mathrm{~S}$ S Dhillon, M S Vitiello, E H Linfield, A G Davies, Matthias C Hoffmann, John Booske, Claudio Paoloni, M Gensch, P Weightman, G P Williams, E Castro-Camus, D R S Cumming, F Simoens, I Escorcia-Carranza, J Grant, Stepan Lucyszyn, Makoto Kuwata-Gonokami, Kuniaki Konishi, Martin Koch, Charles A Schmuttenmaer, Tyler L Cocker, Rupert Huber, A G Markelz, Z D Taylor, Vincent P Wallace, J Axel Zeitler, Juraj Sibik, Timothy M Korter, B Ellison, S Rea, P Goldsmith, Ken B Cooper, Roger Appleby, D Pardo, P G Huggard, V Krozer, Haymen Shams, Martyn Fice, Cyril Renaud, Alwyn Seeds, Andreas Stöhr, Mira Naftaly, Nick Ridler, Roland Clarke, John E Cunningham, and Michael B Johnston, "The 2017 terahertz science and technology roadmap," Journal of Physics D: Applied Physics 50, 043001 (2017)

${ }^{2}$ Tadao Nagatsuma, Guillaume Ducournau, and Cyril C. Renaud, "Advances in terahertz communications accelerated by photonics," Nature Photonics 10, 371-379 (2016).

${ }^{3}$ Wojciech Knap, Mikhail Dyakonov, Dominique Coquillat, Frederic Teppe, Nina Dyakonova, Jerzy Lusakowski, Krzysztof Karpierz, MacIej Sakowicz, Gintaras Valusis, Dalius Seliuta, Irmantas Kasalynas, Abdelouahad El Fatimy, Y. M. Meziani, and Taiichi Otsuji, "Field effect transistors for terahertz detection: Physics and first imaging applications," Journal of Infrared, Millimeter, and Terahertz Waves 30, 1319-1337 arXiv:0907.2523

${ }^{4}$ Maris Bauer, Sebastian Boppel, Jingshui Zhang, Adam Rämer, Serguei Chevtchenko, Alvydas Lisauskas, Wolf- gang Heinrich, Viktor Krozer, and Hartmut G. Roskos, "Optimization of the Design of Terahertz Detectors Based on Si CMOS and AlGaN/GaN Field-Effect Transistors," International Journal of High Speed Electronics and Systems 25, 1640013 (20 ${ }^{5}$ Maris Bauer, Adam Ramer, Serguei A. Chevtchenko, Konstantin Y. Osipov, Dovile Cibiraite, Sandra Pralgauskaite, Kwstutis Ikamas, Alvydas Lisauskas, Wolfgang Heinrich, Viktor Krozer, and Hartmut G. Roskos, "A High-Sensitivity AlGaN/GaN HEMT Terahertz Detector with Integrated Broadband Bow-Tie Antenna," IEEE Transactions on Terahertz Science and Technology 9, 430-444 (2019) ${ }^{6}$ M. Bauer, R. Venckevičius, I. Kašalynas, S. Boppel, M. Mundt, L. Minkevičius, A. Lisauskas, G. Valušis, V. Krozer, H. G. Roskos, R Venckevi`, I Ka``, S. Boppel, M. Mundt, A. Lisauskas, G Valu`, V. Krozer, R. Venckevičius, I. Kašalynas, S. Boppel, M. Mundt, L. Minkevičius, A. Lisauskas, G. Valušis, V. Krozer, and H. G. Roskos, "Antenna-coupled field-effect transistors for multi-spectral terahertz imaging up to $4.25 \mathrm{THz}$," Optics Express 22, 19235 (2014)

${ }^{7}$ Lei Liu, Syed M. Rahman, Zhenguo Jiang, Wenjun Li, and Patrick Fay, "Advanced Terahertz Sensing and Imaging Systems Based on Integrated III-V Interband Tunneling Devices," Proceedings of the IEEE 105, 1020-1034 (2017)

${ }^{8}$ S. Rumyantsev, X. Liu, V. Kachorovskii, and M. Shur, "Homodyne phase sensitive terahertz spectrometer," Applied Physics Letters 111, 121105 (2017)

${ }^{9}$ Diana Glaab, Sebastian Boppel, Alvydas Lisauskas, Ullrich Pfeiffer, Erik Öjefors, and Hartmut G. Roskos, "Terahertz heterodyne detection with silicon field-effect transistors," Applied Physics Letters 96, 042106 (2010).

${ }^{10}$ Leonardo Vicarelli, Miriam Vitiello, Dominique Coquillat, Antonio Lombardo, A.C. Ferrari, W Knap, M Polini, V Pellegrini, and Alessandro Tredicucci, "Graphene field effect transistors as room-temperature terahertz detectors," Nature materials 11, 865-71 (2012)

${ }^{11}$ Leonardo Viti, Jin Hu, Dominique Coquillat, Wojciech Knap, Alessandro Tredicucci, Antonio Politano, and Miriam Serena Vitiello, "Black phosphorus terahertz photodetectors," Advanced Materials 27, 5567-5572 (2015).

${ }^{12}$ Leonardo Viti, Dominique Coquillat, Antonio Politano, Konstantin A. Kokh, Ziya S. Aliev, Mahammad B. Babanly, Oleg E. Tereshchenko, Wojciech Knap, Evgueni V. Chulkov, and Miriam S. Vitiello, "Plasma-wave terahertz detection mediated by topological insulators surface states," Nano Letters 16, 80-87 (2016)

${ }^{13}$ Igor A. Gayduchenko, Maxim V. Moskotin, Yakov E. Matyushkin, Maxim G. Rybin, Elena D. Obraztsova, Victor I. Ryzhii, Gregory N. Goltsman, and Georgy E. Fedorov, "The detection of sub-terahertz radiation using graphene-layer and graphene-nanoribbon FETs with asymmetric contacts," Materials Today: Proceedings 5, 27301-27306 (2018)

${ }^{14}$ Leonardo Viti, Antonio Politano, Kai Zhang, and Miriam Serena Vitiello, "Thermoelectric terahertz photodetectors based on selenium-doped black phosphorus flakes," Nanoscale 11, 1995-2002 (2019)

${ }^{15}$ Gregory Auton, Dmytro B But, Jiawei Zhang, Ernie Hill, Dominique Coquillat, Christophe Consejo, Philippe Nouvel, Wojciech Knap, Luca Varani, and Frederic Teppe, "Terahertz Detection and Imaging Using Graphene Ballistic Rectifiers," Nano Letters 17, 7015-7020 (2017).

${ }^{16}$ Igor Gayduchenko, S. G. Xu, Georgy Alymov, Maxim Moskotin, Ivan Tretyakov, Takashi Taniguchi, Kenji Watanabe, Gregory Goltsman, Andre K. Geim, Georgy Fedorov, Dmitry Svintsov, and Denis A. Bandurin, "Tunnel field-effect transistors for sensitive terahertz detection," Nature Communications 12, 543 (2021), arXiv:2010.03040

17 W Knap, J. Lusakowski, T. Parenty, S. Bollaert, A. Cappy, (2Q09) V. Popov, and M. S. Shur, "Terahertz emission by plasma waves in $60 \mathrm{~nm}$ gate high electron mobility transistors," Applied Physics Letters 84, 2331-2333 (2004). 
${ }^{18}$ V. M. Muravev and I. V. Kukushkin, "Plasmonic detector/spectrometer of subterahertz radiation based on two-dimensional electron system with embedded defect," Applied Physics Letters 100, 10-13 (2012).

${ }^{19}$ Denis A. Bandurin, Dmitry Svintsov, Igor Gayduchenko, Shuigang G. Xu, Alessandro Principi, Maxim Moskotin, Ivan Tretyakov, Denis Yagodkin, Sergey Zhukov, Takashi Taniguchi, Kenji Watanabe, Irina V. Grigorieva, Marco Polini, Gregory N. Goltsman, Andre K. Geim, and Georgy Fedorov, "Resonant terahertz detection using graphene plasmons," Nature Communications 9, 5392 (2018)

${ }^{20}$ Fengnian Xia, Thomas Mueller, Yu Ming Lin, Alberto Valdes-Garcia, and Phaedon Avouris, "Ultrafast graphene photodetector," Nature Nanotechnology 4, 839-843 (2009) arXiv:0912.4794

${ }^{21}$ Xuetao Gan, Ren Jye Shiue, Yuanda Gao, Inanc Meric, Tony F. Heinz, Kenneth Shepard, James Hone, Solomon Assefa, and Dirk Englund, "Chip-integrated ultrafast graphene photodetector with high responsivity," Nature Photonics 7, 883-887 (2013)

${ }^{22}$ Sebastián Castilla, Bernat Terrés, Marta Autore, Leonardo Viti, Jian Li, Alexey Y. Nikitin, Ioannis Vangelidis, Kenji Watanabe, Takashi Taniguchi, Elefterios Lidorikis, Miriam S. Vitiello, Rainer Hillenbrand, Klaas Jan Tielrooij, and Frank H.L. Koppens, "Fast and Sensitive Terahertz Detection Using an Antenna-Integrated Graphene pn Junction," Nano Letters 19, 2765-2773 (2019), 1905.01881

${ }^{23}$ Mikhail Dyakonov and Michael Shur, "Detection, mixing, and frequency multiplication of terahertz radiation by two-dimensional electronic fluid," IEEE Transactions on Electron Devices 43, 380-387 (1996)

${ }^{24}$ W. Knap, F. Teppe, Y. Meziani, N. Dyakonova, J. Lusakowski, F. Boeuf, T. Skotnicki, D. Maude, S. Rumyantsev, and M. S. Shur, "Plasma wave detection of sub-terahertz and terahertz radiation by silicon field-effect transistors," Applied Physics Letters 85, 675-677 (2004)

${ }^{25}$ D. A. Bandurin, I. Gayduchenko, Y. Cao, M. Moskotin, A. Principi, I. V. Grigorieva, G. Goltsman, G. Fedorov, and D. Svintsov, "Dual origin of room temperature subterahertz photoresponse in graphene field effect transistors," Applied Physics Letters 112, 141101 (2018)

${ }^{26}$ Audrey Zak, Michael A. Andersson, Maris Bauer, Jonas Matukas, Alvydas Lisauskas, Hartmut G. Roskos, and Jan Stake, "Antenna-integrated $0.6 \mathrm{thz}$ fet direct detectors based on cvd graphene," Nano Letters 14, 5834-5838 (2014)

${ }^{27}$ Alvydas Lisauskas, Ullrich Pfeiffer, Erik Ojefors, Peter Haring Bolivar, Diana Glaab, and Hartmut G. Roskos, "Rational design of high-responsivity detectors of terahertz radiation based on distributed self-mixing in silicon field-effect transistors," Journal of Applied Physics 105, 114511 (2009)

${ }^{28}$ A. El Fatimy, N. Dyakonova, Y. Meziani, T. Otsuji, W. Knap, S. Vandenbrouk, K. Madjour, D. Théron, C. Gaquiere, M. A. Poisson, S. Delage, P. Prystawko, and C. Skierbiszewski, "AlGaN/GaN high electron mobility transistors as a voltage-tunable room temperature terahertz sources,"
Journal of Applied Physics 107, 1-5 (2010)

${ }^{29}$ Aleksandr S. Petrov and Dmitry Svintsov, "Perturbation theory for two-dimensional hydrodynamic plasmons," Physical Review B 99, 1-8 (2019)

30 Justin C. W. Song, Mark S. Rudner, Charles M. Marcus, and Leonid S. Levitov, "Hot Carrier Transport and Photocurrent Response in Graphene," Nano Letters 11, 4688-4692 (2011) arXiv:1105.1142

${ }^{31}$ Xinghan Cai, Andrei B. Sushkov, Ryan J. Suess, Mohammad M. Jadidi, Gregory S. Jenkins, Luke O. Nyakiti, Rachael L. MyersWard, Shanshan Li, Jun Yan, D. Kurt Gaskill, Thomas E. Murphy, H. Dennis Drew, and Michael S. Fuhrer, "Sensitive roomtemperature terahertz detection via the photothermoelectric effect in graphene," Nature Nanotechnology 9, 814-819 (2014)

${ }^{32}$ M. Sakowicz, M. B. Lifshits, O. A. Klimenko, F. Schuster, D. Coquillat, F. Teppe, and W. Knap, "Terahertz responsivity of field effect transistors versus their static channel conductivity and loading effects," Journal of Applied Physics 110, 054512 (2011)

${ }^{33}$ Shaffique Adam, E. H. Hwang, V. M. Galitski, and S. Das Sarma, "A self-consistent theory for graphene transport," Proceedings of the National Academy of Sciences 104, 18392-18397 (2007) arXiv:0705.1540

${ }^{34}$ A. Sanchez, C. F. Davis, K. C. Liu, and A. Javan, "The MOM tunneling diode: Theoretical estimate of its performance at microwave and infrared frequencies," Journal of Applied Physics 49, 5270-5277 (1978)

${ }^{35}$ Alessandro Principi, Denis Bandurin, Habib Rostami, and Marco Polini, "Pseudo-euler equations from nonlinear optics: Plasmon-assisted photodetection beyond hydrodynamics," Phys. Rev. B 99, 075410 (2019)

${ }^{36}$ Victor Ryzhii, "Terahertz plasma waves in gated graphene heterostructures," Japanese Journal of Applied Physics, Part 2: Letters 45, 923-925 (2006)

${ }^{37}$ T. J. Echtermeyer, L. Britnell, P. K. Jasnos, A. Lombardo, R. V. Gorbachev, A. N. Grigorenko, A. K. Geim, A. C. Ferrari, and K. S. Novoselov, "Strong plasmonic enhancement of photovoltage in graphene," Nature Communications 2, 455-458 (2011)

${ }^{38}$ Maxim Rybin, Alexander Pereyaslavtsev, Tatiana Vasilieva, Vladimir Myasnikov, Igor Sokolov, Alexandra Pavlova, Ekaterina Obraztsova, Andrey Khomich, Viktor Ralchenko, and Elena Obraztsova, "Efficient nitrogen doping of graphene by plasma treatment," Carbon 96, 196-202 (2016)

${ }^{39}$ Maxim G. Rybin, Vera R. Islamova, Ekaterina A. Obraztsova, and Elena D. Obraztsova, "Modification of graphene electronic properties via controllable gas-phase doping with copper chloride," Applied Physics Letters 112, 033107 (2018)

${ }^{40}$ Xuesong Li, Yanwu Zhu, Weiwei Cai, Mark Borysiak, Boyang Han, David Chen, Richard D. Piner, Luigi Colombo, and Rodney S. Ruoff, "Transfer of large-area graphene films for high-performance transparent conductive electrodes," Nano Letters 9, 4359-4363 (2009)

${ }^{41}$ Tony Low, Vasili Perebeinos, Raseong Kim, Marcus Freitag, and Phaedon Avouris, "Cooling of photoexcited carriers in graphene by internal and substrate phonons," Physical Review B 86, 045413 (2012). 